\title{
Acupuntura como recurso terapêutico na dor e na gravidade da desordem temporomandibular
}

\section{Acupuncture as therapeutic resource in the pain and in the severity of the temporomandibular disorder}

\author{
Graciele da Silva Borin ${ }^{1}$, Eliane Castilhos Corrêa ${ }^{2}$, Ana Maria Toniolo da Silva³, Jovana Moura Milanesi ${ }^{1}$
}

Estudo desenvolvido no Laboratório de Motricidade Orofacial da Universidade Federal de Santa Maria (UFSM) - Santa Maria (RS), Brasil.

${ }^{1}$ Mestranda do Programa de Pós-Graduação em Distúrbios da Comunicação Humana da Universidade Federal de Santa Maria (UFSM) - Santa Maria (RS), Brasil.

2 Professora adjunta do Departamento de Fisioterapia e Reabilitação e do Programa de Pós-Graduação em Distúrbios da Comunicação Humana da UFSM - Santa Maria (RS), Brasil.

3 Professora adjunta

do Departamento de Fonoaudiologia e do Programa de Pós-Graduação em Distúrbios da Comunicação Humana da UFSM - Santa Maria (RS), Brasil.

ENDERECO PARA CORRESPONDENCIA

Graciele da Silva Borin - Rua Dr. Pantaleão, 377 - Centro - CEP: 97010-180 - Santa Maria (RS), Brasil - E-mail: graciborin@hotmail.com

\section{APRESENTAÇÃO ago. 2010 \\ ACEITO PARA PUBLICAÇÃO mai. 2011 \\ FONTE DE FINANCIAMENTO nenhuma \\ CONFLITO DE INTERESSES nada a declarar}

Parecer de aprovação do Comitê de Ética e Pesquisa da UFSM $n^{\circ} 23081.018541 / 2007$.
RESUMO: Este estudo avaliou o efeito da acupuntura no nível de dor e gravidade da Desordem Temporomandibular (DTM). Participaram dele 40 mulheres entre 20 e 40 anos com DTM diagnosticada pelo Research Diagnostic Criteria (RDC/TMD). A dor foi avaliada pela escala visual analógica e a gravidade da desordem pelos Índices de Disfunção Craniomandibular e de Fonseca. As 20 primeiras participantes foram submetidas a acupuntura duas vezes na semana por cinco semanas ininterruptas e, após o período, foram reavaliadas. Os dados destas participantes constituíram os resultados para o grupo acupuntura. As demais voluntárias receberam o tratamento após o primeiro grupo e seus dados, coletados no início e após cinco semanas sem tratamento, foram utilizados para controle. Na análise estatística foram levados em conta os testes de Wilcoxon para o nível de dor e Índice Craniomandibular e Teste $t$ de Student para o Índice de Fonseca, com nível de significância de 5\%. Houve redução significante no nível de dor $(p=0,000)$ e na gravidade da DTM pelos Índices Craniomandibular $(p=0,004)$ e de Fonseca $(p=0,000)$ após o tratamento. O grupo controle não apresentou melhora. A efetividade da acupuntura foi demonstrada pela melhora no nível da dor e na gravidade da DTM.

DescritoRes: acupuntura; dor facial;articulação temporomandibular.

ABSTRACT: This study assessed the effect of acupuncture on the pain level and severity of the temporomandibular disorder (TMD). Forty women with TMD diagnosed by Research Diagnostic Criteria, from 20 and 40 years old, took part in the study. Pain was assessed by visual analogue scale and severity by the Craniomandibular Dysfunction and Fonseca Indexes. The first 20 volunteers were undergone to acupuncture twice a week for five weeks without interruption, and after they were reassessed. The results of these participants constituted acupuncture group. The other volunteers received the treatment after the first group and their data, collected in the beginning and after five weeks without treatment, were utilized as control. Statistical analysis of the pain and the Craniomandibular Index was carried out by the Wilcoxon test and the Fonseca index by the Student's $t$-test, with significance level of $5 \%$. There was significant reduction in the pain level $(p=0.000)$ and severity of TMD by the Craniomandibular $(p=0.004)$ and Fonseca Indexes $(p=0.000)$ after the treatment. The control group showed no improvement. The effectiveness of the acupuncture was demonstrated by the improvement in the pain and severity of TMD.

KEYwords: acupuncture; facial pain; temporomandibular joint. 


\section{INTRODUCÃO}

A Desordem Temporomandibular (DTM) caracteriza-se por dor sobre a região da articulação temporomandibular, dor e/ou fadiga nos músculos craniocervicofaciais, especialmente os mastigatórios, limitação e/ou desvios dos movimentos mandibulares, zumbido e presença de ruídos articulares durante a função ${ }^{1}$. Sua etiologia possui causas multifatoriais, podendo ser originada por associações entre fatores posturais, estruturais e psicológicos ${ }^{2}$.

Pehling et al. ${ }^{3,4}$ concluíram com seu estudo que o Índice de Disfunção Craniomandibular é o protocolo apropriado para determinar a gravidade da DTM, principalmente no pós-tratamento.

Outra ferramenta que permite verificar o grau de gravidade da DTM é o Índice de Fonseca ${ }^{5-7}$, pois é de fácil aplicação e compreensão, eliminando as influências do examinador sobre as respostas.

Atualmente, o tratamento da DTM inclui a educação do paciente, terapia comportamental, fisioterapia (ultrassom, eletro-estimulação transcutânea, laser, exercícios, massagem, mobilizações), acupuntura, placa miorrelaxante, ajustamento oclusal, cirurgia e intervenção medicamentosa ${ }^{8,9}$.

Os benefícios alcançados pela acupuntura na anestesia e no tratamento da dor crônica são conhecidos e aceitos pela comunidade científica por meio de diversos estudos controlados ${ }^{10,11}$. Devido à sua capacidade de atuar em nível de sistema nervoso central, liberando opioides endógenos, constitui-se uma ferramenta importante no manejo de pacientes com DTM.

Em decorrência disto, vários autores citam a acupuntura como alternativa para tratamento da sintomatologia dolorosa da $\mathrm{DTM}^{12-16}$. Assim, este estudo foi proposto para avaliar o efeito da acupuntura no nível dor e na gravidade da DTM.

\section{MÉTODOS}

Participaram deste estudo 40 mulheres, brancas, com idade entre 20 e 40 anos, divididas em dois grupos: acupuntura, que realizou intervenção duas vezes por semana $(n=20)$; e controle, que não realizou tratamento $(n=20)$. Todas relataram dor na região da articulação temporomandibular e músculos mastigatórios e foram encaminhadas pela Disciplina de Oclusão do Curso de Odontologia, concordando em participar do estudo e assinando o Termo de Consentimento Livre e Esclarecido (0281.0.243.000-8). Foram excluídas do estudo as voluntárias que apresentaram ausência de elementos dentários ou faziam algum tipo de tratamento odontológico, fisioterapêutico ou medicamentoso e com histórico de traumas ortopédicos ou má-formação na região facial, além de portadores de doença sistêmica ou reumatológica.

\section{Avaliação}

O diagnóstico de DTM foi realizado pelo instrumento Critério de Diagnóstico para Pesquisa de Desordens Temporomandibulares (RDC-TMD), desenvolvido por Dworkin e Le Resche ${ }^{17}$, que contempla os aspectos físicos e psicológicos da DTM ${ }^{18}$.

A gravidade da DTM foi verificada antes e após o tratamento pelo Índice de Fonseca et al. ${ }^{6,7}$, que consta de dez questões, cujas respostas podem ser "Sim", "Às Vezes" e "Não", com pontuação dez, cinco e zero, respectivamente. A soma dos pontos classifica os indivíduos em: DTM - Ausente (0 a 15 pontos), Leve (20 a 40 pontos), Moderada (45 a 60 pontos) ou Grave (70 a 100 pontos). Ainda avaliou-se o Índice de Disfunção Craniomandibular ${ }^{3,4}$, composto por três subíndices: Muscular, Funcional e Articular. O Índice Muscular compreende o exame dos sítios de dor pela palpação bilateral dos músculos mastigatórios intraorais e extraorais, somando 20 sítios. Para cada resposta positiva atribui-se o valor de 1 e para cada negativa 0 e a soma das respostas é dividida por 20. O Índice Funcional inclui 12 itens referentes à amplitude de movimento mandibular, caracterizando a ausência ou presença de dor, limitação do movimento mandibular e desvio mandibular durante abertura. Para cada resposta positiva atribui-se o valor de 1 e para cada negativa, 0 . A soma das respostas é dividida por 12 . O Índice Articular consiste na mensuração da dor com palpação digital em dois sítios de dor para cada articulação e a observação da presença de ruído em cada uma. A soma das respostas positivas é dividida por oito. Para o cálculo final do Índice Craniomandibular, somam-se as respostas dos três subíndices e divide-se por três. A menor pontuação significa menor gravidade da DTM.

A dor foi avaliada, antes e após o tratamento, pela Escala Visual Analógica (EVA), uma linha horizontal de $10 \mathrm{~cm}$, na qual a dor é pontuada de 0 - ausência total de dor - a 10 - nível máximo de dor ${ }^{18,19}$.

\section{Intervenção}

As 20 primeiras participantes foram submetidas à acupuntura duas vezes na semana por cinco semanas ininterruptas e, após o período, foram reavaliadas. As demais voluntárias foram utilizadas como controle enquanto aguardavam pelo atendimento de fisioterapia.

O tratamento foi realizado com agulhas descartáveis $(0,25 \times 0,15 \mathrm{~mm})$ inseridas nos respectivos pontos com a pele previamente limpa com algodão e álcool etílico 70\%. Durante a aplicação da terapia, cada participante permaneceu completamente relaxada, deitada em uma maca na posição de decúbito dorsal por 30 minutos. A terapia de acupuntura totalizou dez atendimentos. Os pontos selecionados para o tratamento foram os referidos na literatura como pontos para o tratamento da DTM e pontos para ansiedade:

ST7 (xiaguan: situa-se logo abaixo o arco zigomático na chanfradura sigmoide, localizada à frente da ATM); ST5 (daying: na margem anterior do músculo masseter com a margem inferior do corpo da mandíbula); TE17 (yifeng: localizado entre o processo mastóideo e o ramo da mandíbula); EXHN5 (taiyang: situado cerca de 1 cun da depressão posterior ao ponto médio entre a extremidade lateral da sobrancelha e o canto externo do olho); GB3 (shangguan: na região préauricular, em uma depressão junto à margem superior do arco zigomático); GB43 (xiaxu: entre a cabeça do quarto e quinto metatarso); LI4 (hegu: entre o primeiro e segundo metacarpo) e EX HN3 (yntang: entre as extremidades médias das duas sobrancelhas) $)^{10-14}$ (Figuras 1 e 2).

Para análise dos resultados, foram considerados os dados obtidos na avaliação inicial (AV) e na reavaliação após dez sessões de acupuntura ou após cinco semanas nos grupos de estudo e de controle 


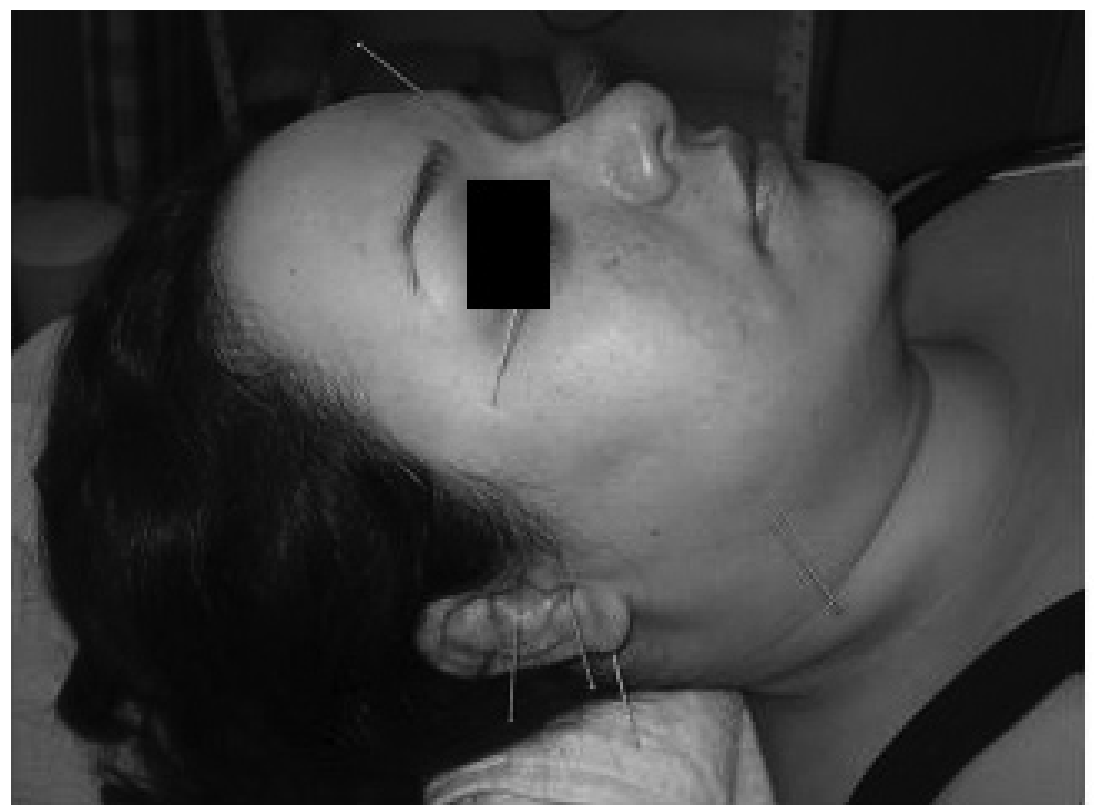

Figura 1. Pontos de acupuntura E7, E5, TA17, VB3, tayang e yntang

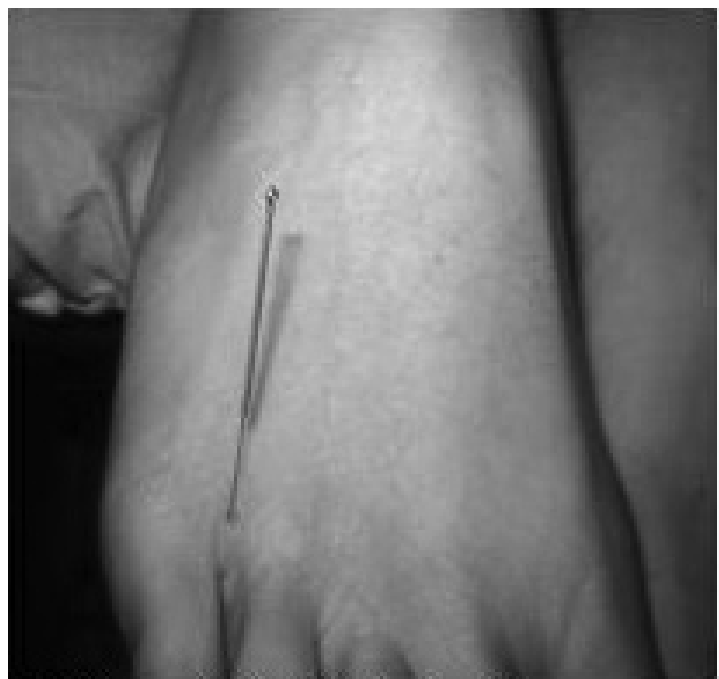

Figura 2. Ponto de acupuntura GB 43 - xiaxu
(R10). A coleta dos dados e a aplicação da acupuntura foram realizadas pelo mesmo pesquisador.

Para análise estatística do nível dor e da gravidade pelo Índice Craniomandibular, como os dados não seguiram uma distribuição normal pelo teste de Lilliefors, utilizou-se o teste não paramétrico de Wilcoxon. A análise estatística da gravidade da DTM avaliada pelo Índice de Fonseca foi calculada pelo Teste $t$ de Student para dados paramétricos. Estabeleceu-se o nível de significância de $5 \%(p<0,05)$.

\section{RESULTADOS}

Verificou-se melhora no nível de gravidade pelo Índice Craniomandibular $(p=0,004)$ e pelo Índice de Fonseca $(p=0,000)$ de indivíduos com DTM após o tratamento por acupuntura (Tabela 1) e no nível de dor $(p=0,000)$ (Tabela 2). Segundo a classificação pelo Índice de Fonseca et al. ${ }^{6}$, antes do tratamento os indivíduos apresentaram a seguinte classificação para DTM: 6 com grau moderado e 14 com grau severo. Após o tratamento, observou-se por esta classificação: 7 com grau leve, 10 com grau moderado e 3 com grau grave (Tabela 3).

\section{DISCUSSÃO}

A partir dos resultados apresentados observou-se que a acupuntura promove redução significante no nível de dor e na gravidade da DTM, o que não foi registrado no grupo controle. Isso também foi evidenciado por Rosted et al. ${ }^{20}$, que verificaram uma significante redução da dor em indivíduos com DTM com base na EVA antes e após o tratamento com

Tabela 1. Resultados referentes à gravidade da desordem temporomandibular (DTM) obtidos pelos índices Craniomandibular e de Fonseca

\begin{tabular}{|c|c|c|c|c|c|c|}
\hline & \multicolumn{2}{|c|}{ Grupo Acupuntura $(n=20)$} & \multirow[b]{3}{*}{$\mathrm{p}$} & \multicolumn{3}{|c|}{ Grupo Controle $(n=20)$} \\
\hline & Pré* & Pós** & & Pré & Pós & \multirow[b]{2}{*}{ p } \\
\hline & M DP & M DP & & M DP & M DP & \\
\hline ICM & $0,73 \pm 0,17$ & $0,60 \pm 0,45$ & $0,004^{*}$ & $1,83 \pm 0,43$ & $2,07 \pm 0,30$ & 0,006 \\
\hline Índice de Fonseca & $74,00 \pm 12,30$ & $49,0 \pm 17,1$ & $0,000 * *$ & $69,75 \pm 16,81$ & $73,00 \pm 18,66$ & 0,163 \\
\hline
\end{tabular}

ICM: Índice Craniomandibular; Pré: Avaliação do grupo acupuntura e grupo controle; Pós: reavaliação após cinco semanas grupo acupuntura e grupo controle DP: desvio padrão; M: média.

*nível de significância $(p<0,05) * *$ nível de significância $(p<0,01)$ 
Tabela 2. Resultados dos valores referentes à mediana e primeiro e terceiro quartis obtidos na avaliação do nível de dor na avaliação inicial (pré) e após cinco semanas (pós).

\begin{tabular}{lllccc}
\hline & & & Q1 & Mediana & Q3 \\
\hline \multirow{4}{*}{ Nível da dor } & \multirow{2}{*}{ GA $(\mathrm{n}=20)$} & Pré & 3,25 & 5,00 & 7,00 \\
& & Pós & $0,00^{*}$ & $0,00^{*}$ & 0,75 \\
& \multirow{2}{*}{ GC $(\mathrm{n}=20)$} & Pré & 3,25 & 5,00 & 7,00 \\
& & Pós & 4,25 & 6,50 & 8,00 \\
\hline
\end{tabular}

GA: grupo acupuntura; GC: grupo controle

*nível de significância $(p<0,001)$

Tabela 3. Classificação da desordem temporomandibular (DTM) segundo o Índice de Fonseca

\begin{tabular}{lcccc}
\hline Classificação Índice & \multicolumn{2}{c}{ Grupo acupuntura $(\mathrm{n}=20)$} & \multicolumn{2}{c}{ Grupo controle $(\mathrm{n}=20)$} \\
\cline { 2 - 5 } de Fonseca & Pré $(\%)$ & Pós $(\%)$ & Pré $(\%)$ & Pós $(\%)$ \\
\hline Ausente & 0 & 0 & 0 & 0 \\
Leve & 0 & 35 & 0 & 0 \\
Moderado & 30 & 50 & 50 & 40 \\
Grave & 70 & 15 & 50 & 60 \\
\hline
\end{tabular}

Ausente: 0-15; Leve: 20-40; Moderada: 45-65; Severa: 70-100

Pré: avaliação do grupo acupuntura e grupo controle; Pós: reavaliação grupo acupuntura e grupo controle após cinco semanas

acupuntura, demonstrando uma redução em $75 \%$ no grau da dor.

Comparando o efeito da acupuntura com a simulação de acupuntura, Smith et al. ${ }^{21}$ se propuseram a estabelecer a verdadeira eficácia deste tratamento. Observaram melhores resultados clínicos, principalmente no nível de dor, avaliada pela EVA para os indivíduos que realmente receberam a acupuntura no ponto E7 durante as três semanas de tratamento. Nosso estudo demonstrou a eficácia da acupuntura, uma vez que o grupo que foi avaliado e reavaliado enquanto aguardava o tratamento não apresentou diferença no nível de dor e gravidade da DTM, enquanto o primeiro atendido teve redução de ambas.

Rosted $^{22}$ realizou uma revisão com 14 artigos sobre o uso da acupuntura em DTM e analisou as metodologias descritas nos estudos, recomendações para o uso da acupuntura na DTM, pontos utilizados, tipo de estimulação, número de sessões e o intervalo entre elas. $\mathrm{O}$ autor concluiu que a acupuntura é uma terapêutica eficaz para o tratamento da DTM quando realizada no mínimo 30 minutos em cada atendimento e em média de 6 a 8 tratamentos. No presente estudo foram realizados 10 atendimentos com duração de 30 minutos cada, demonstrando estar dentro das recomendações sugeridas.

Rodrigues et al. ${ }^{23}$ verificaram, com uso de estimulação elétrica transcutânea (TENS), que o alívio da dor e o relaxamento muscular promovem o restabelecimento da função muscular e sugerem uma provável redução da gravidade da DTM. Ao encontro destes resultados, nosso estudo também demonstrou que apenas a diminuição da dor reduziu a gravidade da DTM, provavelmente devido ao relaxamento muscular.

A dor muscular local é um dos sintomas mais comuns da DTM e, segundo alguns autores ${ }^{24,25}$ essa condição de dor deve-se à isquemia muscular local. A diminuição da microcirculação pode levar à liberação de mediadores químicos que sensibilizam nervos periféricos podendo levar à dor. A acupuntura é uma opção para a DTM, pois produz o aumento do fluxo sanguíneo local e a liberação de opioides endógenos ${ }^{26}$. De acordo com Okada et al. ${ }^{27}$, tratamentos que melhoram o fluxo sanguíneo levam ao relaxamento muscular e são efetivos no alívio da dor muscular em pacientes com DTM. Os achados do presente estudo demonstraram os efeitos benéficos da acupuntura sobre a dor muscular.

A melhora do nível de dor encontrada no presente estudo pode ser também explicada pelo fato da acupuntura agir no eixo hipotálamo-pituitário-adrenal e, assim, por meio de transmissores humorais e neurais, ocorre a ativação de centros corticais bem como a liberação de substâncias anti-inflamatórias e analgésicas, proporcionando o alívio da dor ${ }^{28}$.

A avaliação da gravidade da DTM pelo Índice Craniomandibular utilizado no presente estudo está de acordo com Almeida et al. ${ }^{29}$, que afirmam que este índice permite que a gravidade da desordem seja categorizada de forma individual. Também possibilita examinar a incidência do problema numa população específica, mensurando a efetividade das terapias e auxiliando no estudo dos fatores etiológicos, no diagnóstico das DTM e na conduta frente ao paciente.

Os resultados deste estudo mostraram redução na gravidade da DTM pelos escores dos Índices de Fonseca e Craniomandibular. Neste último, foi reduzido de 0,73 para 0,6 ( $p=0,004)$ após dez sessões de acupuntura. Resultados semelhantes foram observados por Pehling et al. ${ }^{3}$, que utilizaram o Índice Craniomandibular para avaliar pacientes com DTM, os quais apresentaram redução da gravidade, demonstrada pela redução significante do valor do pré para pós-tratamento de 0,41 para 0,29. Cabe salientar o grau mais leve de gravidade de DTM nestes pacientes e que o tratamento utilizado pelos autores constituiu-se de uma combinação aleatória de fisioterapia, intervenção medicamentosa e mudança comportamental.

A acupuntura é um recurso terapêutico que intervém sobre os aspectos psicológicos e físicos do paciente, pela liberação de opioides ${ }^{26}$ e melhora do fluxo sanguíneo ${ }^{27}$, respectivamente, o que pode ter contribuído para o alívio da dor dos pacientes deste estudo. Tal achado concorda com pesquisas que detectaram associação entre DTM e depressão ${ }^{30} \mathrm{e}$ relatam que a dor persistente e recorrente da DTM tem um impacto potencial no cotidiano, principalmente no estado psicológico, além do desconforto, incapacidade física e funcional, o que leva a limitações qualidade de vida ${ }^{31,32}$. 
Há algumas evidências de que, além da acupuntura, os seguintes tratamentos podem também ser eficazes em aliviar a dor da DTM: aparelhos oclusais, terapia comportamental, exercícios mandibulares, treinamento postural e farmacoterapia. Evidência para o efeito das eletroterapias e a cirurgia são insuficientes, e o ajuste oclusal parece não ter efeito ${ }^{8,33}$.

Nosso estudo utilizou somente a acupuntura como forma de tratamento e apresentou resultados satisfatórios na dor e na gravidade, porém a acupuntura pode ser realizada em combinação com outras terapias, como foi observado por Zhou e Zhao ${ }^{34}$, que compararam o efeito terapêutico da acupuntura com a combinação do ultrassom e a eletroterapia em pacientes com DTM. Sessenta e oito pacientes com DTM foram distribuídos de maneira aleatória em dois grupos para receber os dois tratamentos. Houve resultados estatisticamente significantes e melhores para a acupuntura em combinação com o ultrassom. Estes resultados positivos também foram observados por Shin et $\mathrm{al}^{35}$, que investigaram a associação da massagem e a aplicação da acupuntura em indivíduos com DTM e seu efeito sobre a sintomatologia dolorosa e abertura máxima da boca. Os autores obtiveram melhora significante na redução da dor $(p=0,001)$ e aumento da abertura máxima da boca $(p=0,004)$. Assim, os efeitos positivos da acupuntura na DTM podem ser obtidos quando esta é utilizada isoladamente ou combinada a outros recursos terapêuticos.

Os achados deste estudo mostraram que o grupo controle não apresentou diferença entre os nível da dor e da gravidade da DTM entre a avaliação inicial e após cinco semanas. Este resultado foi confirmado em algumas pesquisas ${ }^{8,33}$ que encontraram evidências de que a acupuntura é melhor que técnicas conservadoras e melhor comparável a outras formas de tratamento conservadoras. Mas, devido às deficiências metodológicas, MacPherson et al.9,33 recomendam diretrizes específicas para melhorar a qualidade desta intervenção e, antes de a eficácia da acupuntura ser determinada, mais estudos primários são necessários.

A técnica de acupuntura é indicada no tratamento de fisioterapia por ser de baixo custo, rápida aplicação e não apresentar efeitos colaterais. Além disto, demonstra ser eficaz pela sua abordagem com enfoque tanto sobre os aspectos físicos quanto emocionais presentes na DTM e como coadjuvante de outras terapias.

\section{CONCLUSÃO}

O tratamento de fisioterapia com a técnica de acupuntura demonstrou ser eficaz na diminuição do nível de dor e na gravidade da DTM no grupo acupuntura.

\section{REFERÊNCIAS}

1. Di Fabio RP. Physical therapy for patients with TMD: a descriptive study of treatment, disability, and health status. J Orofac Pain. 1998;12(2):124-35.

2. Pedroni CR, De Oliveira AS, Guaratini MI. Prevalence study of signs and symptoms of temporomandibular disorders in university students. J Oral Rehabil. 2003;30(3):283-9.

3. Pehling J, Schiffman E, Look J, Shaefer J, Lenton P, Fricton J. Interexaminer reliability and clinical validity of the temporomandibular index: a new outcome measure for temporomandibular disorders. J Orofac Pain. 2002;16(4):296-304.

4. Grossi DB, Chaves TC, Oliveira AS. Principais instrumentos para avaliação da disfunção temporomandibular, parte I: índices e questionários; uma contribuição para a prática clínica e de pesquisa. Fisioter. Pesqui. 2008;15(1):92-100.

5. Toledo BAS, Capote TSO, Campos JADB. Associação entre disfunção temporomandibular e depressão. Cienc Odontol Bras. 2008;11(4):75-9.

6. Campos JADB, Gonçalves DAG, Camparis CM, Speciali JG. Confiabilidade de um formulário para diagnóstico da severidade da disfunção temporomandibular. Rev Bras Fisioter. 2009;13(1):38-43.

7. Da Fonseca DM, Bonfante G, Valle AL, Freitas SFT. Diagnóstico pela anamnese da disfunção craniomandibular. Rev Gaúcha Odontol. 1994;4(1):23-32.

8. List T, Axelsson S. Management of TMD: evidence from systematic reviews and meta-analyses. J Oral Rehabil. 2010;37(6):430-51.

9. MacPherson $\mathrm{H}$, White $\mathrm{A}$, Cummings $\mathrm{M}$, Jobst KA, Rose K, Niemtzow RC, et al. Standards for reporting interventions in controlled trials of acupuncture: the recommendations. J Altern Complement Med. 2002;8(1):85-9.

10. Cho SH, Whang WW. Acupuncture for temporomandibular disorders: a systematic review. J Orofac Pain. 2010;24(2):156-62.

11. Wong T. Use of electrostimulation of acupuncture points in general dental practice. Anesth Progr. 1989;36(45):243-4.

12. Nader HA. Acupuntura na odontologia: um novo conceito. Rev Assoc Paul Cir Dent. 2003;57(1):49-51.

13. Kreiner M. La acupuntura como mecanismo de analgesia y anestesia en odontologia: aspectos biológicos y terapéuticos. Odontoestomatol. 1994;5(5):37-43.

14. List T, Helkimo M, Andersson S, Carlsson GE. Acupuncture and occlusal splint therapy in the treatment of craniomandibular disorders. Part I. A comparative study. Swed Dent J. 1992;16(4):125-41. 


\section{Referências (cont.)}

15. Okeson JP. Dor orofacial: guia de avaliação, diagnóstico e tratamento. São Paulo (SP): Quintessence; 1998.

16. Dallanora LJ, Inoue RT, Feltrin PP, Santos VMA. Avaliação do uso da acupuntura no tratamento de paciente com bruxismo. Rev Gaúcha Odontol. 2004;52(5)333-9.

17. Dworkin SF, Le Resche L. Research diagnostic criteria for temporomandibular disorders: review, criteria, examinations and specifications, critique. J Craniomandib Disord. 1992;6(4):301-55.

18. Venezian GC, da Silva MA, Mazzetto RG, Mazzetto MO. Low level laser effects on pain to palpation and electromyographic activity in TMD patients: a doubleblind, randomized, placebo-controlled study. Cranio. 2010;28(2):84-91.

19. Kuttila M, Le Bell Y, Savolainen-Niemi E, Kuttila S, Alanen P. Efficiency of occlusal appliance therapy in secondary otalgia and temporomandibular disorders. Acta Odontol Scand. 2002;60(4):248-54.

20. Rosted P, Bundgaard M, Pedersen AM. The use of acupuncture in the treatment of temporomandibular dysfunction--an audit. Acupunct Med. 2006;24(1):16-22.

21. Smith P, Mosscrop D, Davies S, Sloan P, Al-Ani Z. The efficacy of acupuncture in the treatment of temporomandibular joint myofascial pain: a randomized controlled trial. J Dent. 2007;35(3):259-67.

22. Rosted P. Pratical recommendations for the use of acupuncture in treatment of temporomandibular disorders based on the outcome of published controlled studies. Oral Dis. 2001;7(2):109-15.

23. Rodrigues D, Oliveira AS, Bérzin F. Effect of tens on the activation pattern of the masticatory muscles in TMD patients. Braz J Oral Sci. 2004;3:510-5.

24. Larsson SE, Bodegard L, Henriksson KG, Oberg PA. Chronic trapezius myalgia. Morphology and blood flow studied in 17 patients. Acta Orthop Scand. 1990;61(5):394-8.

25. Tullberg M, Alstergren PJ; Ernberg MM. Effects of lowpower laser exposure on masseter muscle pain and microcirculation. Pain. 2003;105(1-2):89-96.
26. Yamamura Y. Acupuntura tradicional: a arte de inserir. 2. ed. São Paulo (SP): Editora Roca; 2001.

27. Okada K, Yamaguchi T, Minowa K, Inoue N. The influence of hot pack therapyon the blood flow in masseter muscles. J Oral Rehabil. 2005;32(7):480-6.

28.Villalobos YC, Hidalgo SH, Goméz SMD, Navarro IC. Eficacia de la acupuntura en el síndrome dolordisfunción del aparato temporomandibular. Archivo médico de camaguey. 2006;10(5):4.

29. Almeida RAC, Vasconcelos BCE, Cunha SC, Nogueira RVB, Duarte AP. Índices de Helkimo e Craniomandibular para diagnóstico de desordens têmpero-madibulares: revisão de literatura. Rev Cir Traumatol Buco-Maxilo-Fac. Camaragibe. 2005;5(3):9-16.

30. Basso D, Corrêa E, da Silva AM. Efeito da reeducação postural global no alinhamento corporal e nas condições clínicas de indivíduos com disfunção temporomandibular associada a desvios posturais. Fisioter Pesqui. 2010;17(1):63-8.

31. Selaimen C, Brilhante DP, Grossi ML, Grossi PK. Depression and neuropsychology testing in patients with temporomandibular disorders. Cien Saude Colet. 2007;12(6):1629-39.

32. Rancan SV, Bataglion C, Bataglion SA, Bechara OM, Semprini M, Siéssere $S$, et al. Acupuncture and temporomandibular disorders: a 3-month follow-up EMG study. J Altern Complement Med. 2009; 15(12):1307-10.

33. Schiffman EL, Truelove EL, Ohrbach R, Anderson GC, John MT, List T. The research diagnostic criteria for temporomandibular disorders. I: overview and methodology for assessment of validity. J Orofac Pain. 2010;24(1):7-24.

34. Zhou FH, Zhao HY. Acupuncture and ultrasound therapy for temporomandibular disorders. Di Yi Jun Yi Da Xue Xue Bao. 2004;24(6):720-1.

35. Shin BC, Ha CH, Song YS, Lee MS. Effectiveness of combining manual therapy and acupuncture on temporomandibular joint dysfunction: a retrospective study. Am J Chin Med. 2007;35(2):203-8. 